\title{
Thorium fuelled reactors - do they need an accelerator?
}

\section{Roger Barlow*}

The University of Huddersfield

E-mail: R.Barlowdhud.ac.ur

We present and evaluate the arguments put forward for Accelerator Driven Subcritical Reactor systems, especially in the context of thorium fuel. Some advocates of thorium fuel argue that an accelerator is not necessary, others that it is. We consider the arguments and find that although several of the reasons commonly put forward do not stand up to robust scrutiny, an accelerator is necessary to achieve stable operation of a thorium breeder reactor, avoiding cyclic behaviour in the reactivity which would make operation of an accelerator-free design unstable.

4th Workshop on ADS and thorium

31 August - 2 September 2016

University of Huddersfield, England

\footnotetext{
* Speaker.
} 


\section{Introduction}

Reactors may be critical or subcritical: they may use uranium or thorium as the basis for their fuel. Yet since Rubbia's original proposal [四, thorium and ADSRs have generally been paired[】, [3]. This is not universal: there are proposals for uranium-fuelled ADSRs such as MYRRHA [䧃], and for the use of thorium in conventional [[]] or molten salt reactors [ [6]. But there has usually been a link between the two.

The argument is made that although the costs of the accelerator, in both construction and performance, are not small - typically $10-20 \%$ of the total - they are outweighed by the benefits. But this is not universally accepted. Some deny that such benefits exist at all. Kirk Sorensen famously commented [ [ $[$ ] that "A thorium reactor needs an accelerator like a fish needs a bicycle." Those advocating ADSR systems need to respond by presenting the arguments, or we will be accused of being accelerator builders looking for an excuse to build unnecessary machines. And we must do so honestly, as objective scientists rather than political advocates. This paper is an attempt to form and present such an argument.

\section{Analysis of some common arguments for including an accelerator}

We first examine the arguments that have been used in favour of ADSR systems, for both uranium and thorium systems

\subsection{Safety}

When the accelerator in an ADSR is switched off the fission reactions cease. There is no possibility of a criticality incident, as happened at Chernobyl. This is a simple and powerful argument which the general public can appreciate.

But it is hardly rigorous. Modern reactors have negative reactivity coefficients. A design like the RBMK, with a positive void coefficient, is now unthinkable - the nuclear industry can learn from past mistakes. ADSRs are safe in this respect - but so is any reactor running today.

When the earthquake struck at Fukushima-Daiichi, three of the six reactors were switched off, and the other three shut down smoothly. There was no criticality incident. Problems came from the continuing heat generated by fission products, and this heat will also occur for ADSRs. It is true that most designs would be safe as they use a liquid coolant (lead, lead-bismuth, or molten salt) which continues to circulate by convection even if power is lost, but this feature is not directly linked to the accelerator.

It may be that the public will be more prepared to accept a system with a clear capability of being switched off (the "big red switch"), but this is not really a sound reason for adding an accelerator to a reactor.

\subsection{Neutron production}

A typical fission produces an average $v$ neutrons - the value varies with the target and the neutron energy, but is typically about 2.5. These are divided into $n_{f}$ neutrons causing further fission, $n_{b}$ absorbed by a fertile nucleus which subsequently produces a fissile one, and $n_{c}$ which 
are absorbed in the control rods, by fission products, or in other non-productive reactions, or escape from the reactor entirely.

$$
n_{f}+n_{b}+n_{c}=v
$$

A critical reactor has to ensure that $n_{f}=1$. This leaves $\sim 1.5$ to make up $n_{b}+n_{c}$, which gives considerable flexibility to the design..

A stable critical breeder reactor also requires $n_{b}=1$, so that burnt fuel nuclei are replaced. This constrains the design, as only $v-2$ neutrons can be lost. The original Energy Amplifier proposal[ [W] gives $v=2.3$ for fast neutrons in thorium, though other references[[] give a more comfortable $v=2.48-2.55$. Without enough neutrons a chain reaction is not sustainable, so the reactor is subcritical and must be operated as an amplifier for other neutrons from a separate source.

In qualitative terms this is plausible, but in quantitative terms most designs operate with a criticality $k_{\text {eff }}$ in the range of 0.95 to 0.98 (or even 0.995 [8]]). Only $2 \%$ to $5 \%$ of the neutrons come from spallation. If a reactor has a criticality in the high nineties, the design can probably be adjusted slightly to make it up to the full 1.0. The numbers have to be considered properly in any case, but it is implausible that such a small change would not be possible - particularly if the accelerator and target can be removed.

So again the argument is weak. If the neutron deficit is small it can probably be fixed. If the neutron deficit is large then a large accelerator is needed, with costs much greater than the usually quoted $10-15 \%$.

\subsection{Fast spallation neutrons are needed for incineration}

Production of Minor Actinide (MA) waste is undesirable and should be minimised, or if possible eliminated. For these problematic nuclei (list??) the fission cross sections are low for thermal neutrons, but high for fast neutrons. Neutrons from spallation are faster than neutrons from fission, and are needed for a reactor that will consume MA waste from other reactors in addition to its own.

While it is true that spallation neutrons are faster than fission neutrons, this difference is not large, particularly after the neutrons have undergone elastic collisions with target nuclei (for a discussion of the spallation spectrum see [Q]]). Nor is it particularly significant. Fission probabilities increase significantly between thermal energies and $\sim 1 \mathrm{MeV}$, but not much between 1 and $10 \mathrm{MeV}$.

Furthermore, as noted in the previous section, in an ADSR the spallation neutrons are only a small fraction of the total. Any advantageous increase in cross section affects only 2-5\% of the neutrons, and any effect is washed out. Indeed studies have found no advantage in the spallation neutron spectrum [미].

\subsection{Spallation can give a very intense neutron flux}

Bowman[ए] proposed the use of a spallation source to generate a very high intensity of neutrons: $10^{16}$ neutrons $/ \mathrm{cm}^{2} / \mathrm{s}$ instead of the more usual $10^{14}$. This was for the purpose of incinerating problematic MA nuclei which have already survived months in the reactor and clearly need something stronger. He pointed out that, for example, ${ }^{237} \mathrm{~Np}$ will absorb a neutron to become ${ }^{238} \mathrm{~Np}$ which in a normal reactor decays to ${ }^{238} \mathrm{Pu}$, which then requires typically two further neutron ab- 


\begin{tabular}{|c|c|}
\hline Nucleus & Delayed neutron Fraction $\xi$ \\
\hline${ }^{233} \mathrm{U}$ & $2.7 \times 10^{-3}$ \\
${ }^{235} \mathrm{U}$ & $6.5 \times 10^{-3}$ \\
${ }^{239} \mathrm{Pu}$ & $2.1 \times 10^{-3}$ \\
${ }^{239} \mathrm{Pu}$ & $2.1 \times 10^{-3}$ \\
${ }^{241} \mathrm{Am}$ & $1.3 \times 10^{-3}$ \\
\hline
\end{tabular}

Table 1: Delayed neutron fractions for fissile nuclei. Taken from [[13]

sorptions to fission. But a high flux can cause the intermediate ${ }^{238} \mathrm{~Np}$ to fission, shortening the process and contributing positively rather than negatively to the reactivity.

But, quite apart from the fact that high flux reactors are possible, this is a proposal for a specific waste management problem, for thermal reactors. It does not generalise to the overall incineration problem, and certainly not to energy generation, and does not apply to fast neutrons. In particular it is inappropriate for thorium, as a high flux increases the probability that the intermediate ${ }^{233} \mathrm{~Pa}$ isotope suffers neutron absorption rather than decay to to a fuel nucleus. It would be taking it out of context to use it as a general argument in favour of ADSR systems.

\subsection{To overcome problems with minor actinides}

The justification for the MYRRHA proposal [ए2] is given as

Critical reactors loaded with fuel containing large amounts of minor actinides pose safety problems caused by unfavorable reactivity coefficients and small delayed neutron fractions.

A reactor requires some number of the $v$ fission neutrons to be 'delayed', or it will respond to changes in criticality, from the control rods or other changes, with the time constant of the mean lifetime of the neutrons. (This is shorter for fast neutrons than for thermal neutron, but this is not an issue: a millisecond is as dangerous as a microsecond in this context.). If the criticaiity of a reactor, due to control rod movement or fuel evolution, varies from 1, the operators/control system can maintain stable operation by applying an opposite change within the delayed neutron timescale (typically many seconds) provided the prompt criticality limit is not exceeded. But control rods cannot be adjusted with infinite precision: if this band becomes too narrow then safe operation is impossible.

The numbers of delayed neutrons for important fissile nuclei is shown in table $\mathrm{W}$.

The fraction for the ${ }^{233} \mathrm{U}$ used in thorium reactors is somewhat below that for ${ }^{235} \mathrm{U}$, which is presumably safe and regarded as standard, but above that for ${ }^{239} \mathrm{Pu}$, which is also in use. However the figures for MA nuclei are smaller - in some cases much smaller - and it is not surprising that a study of an MA loaded reactor judged it to be 'unsafe for critical operation' [14]

But the delayed neutron fraction in a reactor is the weighed average over all the relevant nuclei, and if the admixture of MA is small, the effective $\xi$ will not change greatly. A moderate amount (5-10\%) of MA waste would presumably be added to the reactor fuel without making it unsafe. The reactor in the study [14] was loaded with 35\% MA mix. So this may justify the use of ADSRs in dedicated reactors set up to incinerate waste from many standard reactors, but does not preclude the operation of a fast thorium reactor as an incinerator as well as a power source, provided one its not too ambitious. 
Figures for Minor Actinide temperature reactivity coefficients - the loss in reactivity due to increased absorption as the Doppler effect widens resonances with increasing temperature - are hard to find. One study [15]] quotes negative values for them, but says that Minor Actinides cause a positive coolant void coefficient, which can be circumvented by using a small reactor core to increase edge losses. Again, they consider fuel mixtures with very high ( 50\%) MA content, and they find operation of a reactor possible even at this level. If there are problems with the reactivity properties, they can be managed by using a modest fraction of MA in the fuel

\section{The ideal reactor and its operation}

Consider an ideal thorium reactor. We suppose that the problems of startup have been overcome in some way, and that the fuel rods contain a mixture of ${ }^{232} \mathrm{Th}$ and ${ }^{233} \mathrm{U}$. The fuel element lifetime is long as the fertile to fissile conversion counteracts the poisoning by fission products. It operates as a fast reactor, burning its own MA waste with some spare capacity .

We would also like it to be able to vary its power output, to accommodate variations in demand and supply from other sources.

This will lead to changes in operating conditions, on top of those due to fuel evolution (slow) and refuelling (rare). Any such changes in power are coupled to the neutronics. The nuclear cycle runs

$$
\text { Fission neutrons }+{ }^{232} \mathrm{Th} \rightarrow{ }^{233} \mathrm{Th} \rightarrow{ }^{233} \mathrm{~Pa} \rightarrow{ }^{233} \mathrm{U} \rightarrow \text { fission neutrons }
$$

and the power generated is given by the number of such cycles that are taking place in the reactor: at a higher or lower power all rates increase or decrease.

But when the power is increased, all the rates change except one: the ${ }^{233} \mathrm{~Pa} \rightarrow{ }^{233} \mathrm{U}$ process. The rate of ${ }^{233} \mathrm{~Pa}$ formation increases as more neutrons are captured by the thorium, but the 27 day half life means that the ${ }^{233} \mathrm{U}$ formation continues at the old rate. It only rises to the new value asymptotically. (The half life of the ${ }^{233} \mathrm{Th}$ is much shorter, at 22 minutes, and the effect can be ignored.)

We can model this simply, in terms of the densities $\rho_{U}$ and $\rho_{P a}$ and the neutron numbers $n_{b}$ and $n_{c}$ introduced earlier. We ignore the variation in $\rho_{t h}$, and also the effect of fission products, as these operate on a long timescale. Suppose the power is fixed at $N$ fissions/volume/second. The densities obey

$$
\begin{aligned}
& \frac{d \rho_{P a}}{d t}=N n_{b}-\lambda \rho_{P a} \\
& \frac{d \rho_{U}}{d t}=-N+\lambda \rho_{P a}
\end{aligned}
$$

If power is increased, ${ }^{233} \mathrm{U}$ fuel is consumed at the new rate but replaced at the old rate, the fuel concentration falls and the criticality drops. Likewise if the power is decreased, the fuel concentration rises and the reactor criticality increases. The operator (we will refer to 'the operator' though this system will be automatic) will adjust the control rods to remedy this, changing $n_{c}$ to ensure that $n_{f}$ stays fixed at exactly 1 . 
But changes in $n_{c}$ also affect $n_{b}$. If the fuel density $\rho_{U}$ drops, decreasing the probability of fission, the rods are withdrawn so that fewer neutrons are lost to absorption. But the extra neutrons are then available not just for fission but also for breeding. If $n_{c}$ is reduced then $n_{f}$ and $n_{b}$ both rise.

So an increase in power tends to lower the fuel density, which is compensated by a withdrawal of control rods, which leads to an increase ${ }^{233} \mathrm{~Pa}$ formation. The accumulated excess of ${ }^{233} \mathrm{~Pa}$ works through the system with a 27 day half-life, leading to a state in which the reactor has a high fuel density, requiring the insertion of the control rods. The system will oscillate.

Assuming that the control rods are varied to keep the number of fission neutrons constant leads to

$$
\frac{d n_{c}}{d \rho_{f}}=-\frac{d n_{b}}{d \rho_{f}}=\frac{n_{b}}{\rho_{f}}
$$

To see the effect, we have simulated a simple system which generates $500 \mathrm{MW}$ thermal power, assuming that it starts after a period of downtime with no protactinium, but a uranium density sufficient to make it critical. The evolution wth time of the fuel density is shown in Figure 1, and Figure 2 shows the number of neutrons that are lost or captured (i.e. not used for fission or breeding).

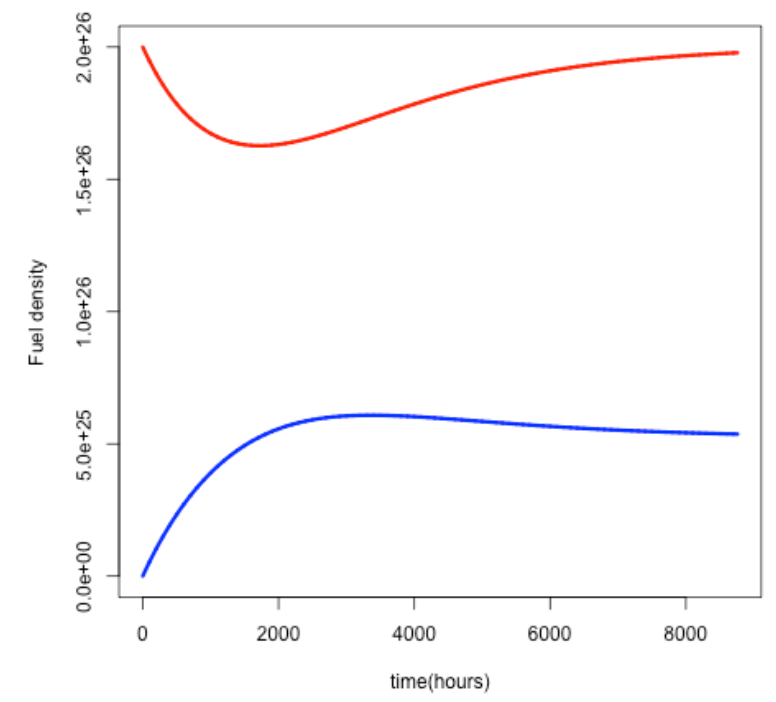

Figure 1: Variations in fuel density: the blue is protactinium and the red is uranium

It can be seen that the oscillation is slow and large. Given the inevitable neutron losses to it is questionable whether the design is viable. The margin to cover absorption, escape etc is not 0.5 neutrons but only $\sim 0.25$.

This is an arbitrary example and not necessarily typical. But stable operation requires $n_{f}=$ $n_{b}=1$. Wiithout an accelerator there is only one parameter to adjust ( $n_{c}$, via the control rods) and this must be used to keep $n_{f}=1$, so that $n_{b}$ will vary. This leads to changes in the fuel density which pushes further changes to $n_{f}$, requiring further changes to $n_{c}$. These changes may be large, and may use up all the surplus neutrons, killing the chain reaction. 


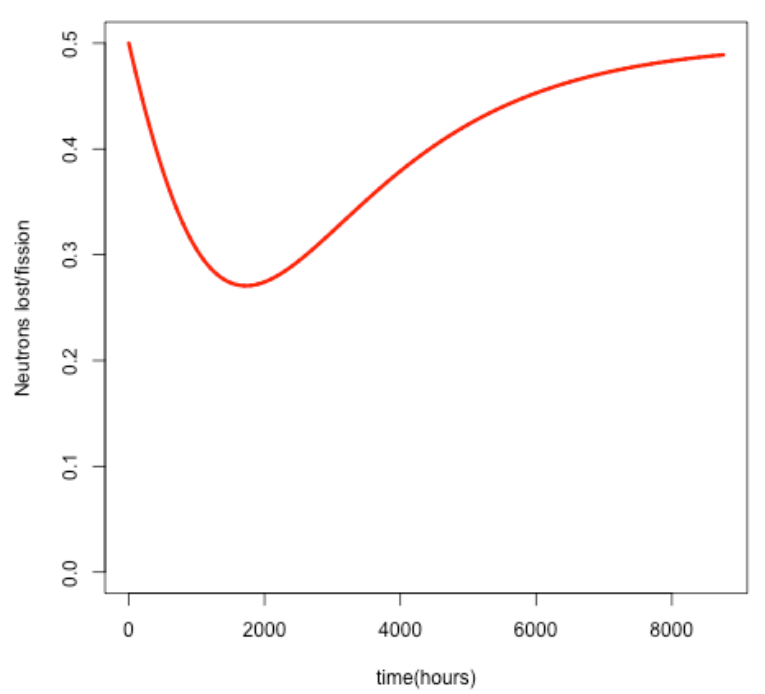

Figure 2: Variations in the number of 'spare' neutrons $n_{c}$

\section{Some considerations}

Having established that a stable $n_{f}=n_{b}=1$ thorium reactor cannot be achieved raises some immediate questions.

\subsection{Breeder reactors are not impossible}

Breeder reactors have operated successfully. (They have had problems, but for different reasons). But they have operated with uranium/plutonium fuel rather than thorium, and the ${ }^{239} \mathrm{~Np}$ state which is the analogue of ${ }^{233} \mathrm{~Pa}$ has a half life of only the new rate but replaced at the old rate, the fuel concentration falls and the criticality drops. Likewise if the power is decreased, the fuel concentration rises and the reactor criticality increases. The o 2.36 days, so the oscillations are much more rapid and do not have time to build up. Furthermore they have operated by extraction and re-processing of the fuel, not the long term breeding within a single fuel rod that enables thorium reactors to operate stably over periods of the order of years.

\subsection{Does an accelerator cure the problem?}

Clearly having two adjustable parameters - the accelerator power and the control rods - gives more control over the reactor. In operation the beam current is adjusted to determine the power level, and the control rods ensure that $n_{b}=1$.

Alternatively one can (if one is confident of the design) operate an ADSR without control rods, and ride out the variations in reactivity. If the criticality of an ADSR is lower than planned, the beam current can be increased, and even if that is not possible the worst that happens is that the reactor operates at a lower power than hoped for, whereas if the criticality of a conventional reactor is lower than planned, it delivers nothing. 


\subsection{Are there alternative solutions?}

There may be other possible ways of dealing with these reactivity cycles. The neutron flux in the core is higher in the centre than at the edges, and this effect can be increased by the design, if desired. High flux regions will see decreases in $\rho_{U}$, whereas low flux regions will see increases. Interchanging fuel rods between low flux and high flux regions could provide an additional degree of control. This could also be achieved in a molten salt reactor, using the flow of the fuel mixture between different regions.

One can even envisage a system in which fuel rods spend a short time in a high flux area, increasing $\rho_{P a}$ while $\rho_{U}$ falls, and are then removed from the reactor entirely for $\sim 27$ days . More drastically still, fuel rods could be opened and the uranium chemically separated for reintroduction to the core.

How this can be achieved - if at all - lies beyond the scope of this paper. The relevant point here is that it would add a degree of complexity to the operation of the reactor which is at least commensurate with the addition of an accelerator.

\section{Conclusions}

Even though some arguments commonly used in favour of ADSRs may be weak, thorium reactors do need an accelerator - or, at least, an accelerator would bring great benefits, enabling the long lifetime of the fuel rods to be exploited with stable operation in a load-following power generating system, without the oscillations in reactivity due to the impossibility of breeding at a constant rate in a simple reactor.

\section{References}

[1] F Carminati et al.. An Energy Amplifier For Cleaner And Inexhaustible Nuclear Energy Production Driven By a Particle Accelerator, CERN report CERN/AT/93-47

[2] H. Nifenecker, O. Meplan and S. David Accelerator Driven Subcritical Reactors, IoP Publishing (2003)

[3] S Peggs et al., Thorium Energy Futures, Proc. IPAC2012, New Orleans, USA, MOBBA01 (2012)

[4] H. Aït Abderrahim, P Baeten, D De Bruyn, J Heyse, P Schuurmans, J Wagemans MYRRHA, a Multipurpose hYbrid Research Reactor for High-end Applications, Nuclear Physics News, 2024 (2010)

[5] A Radkowsky and A Galperin The Nonproliferative Light Water Thorium Reactor: a New Approach to Light Water Reactor Core Technology, Nucl. Technol 124215 (1998)

[6] H G MacPherson ,The Molten Salt Reactor Adventure Nuclear Science and Engineering 90, 374 (1985)

[7] http://thoriummsr.com/2011/06/what-garbage-a-thoriumreactor-needs-an-accelerator-like-a-fish-needs-a-bicycle/ Accessed $16 / 3 / 17$

[8] V Ashley and R Ashworth, The Technically Viable ADTR ${ }^{T M}$ Power Station Nuclear Future 7, 3 (2011)

[9] A Rummana, these proceedings 
[10] Maurcio Gilberti et al., Transuranics Transmutation Using Neutrons Spectrum from Spallation Reactions, Science and Tech. of Nucl. Installations, 104739, (2015)

[11] C D Bowman et al., Nuclear energy generation and waste transmutation using an accelerator-drive intense thermal neutron source Nucl. Instr. \& Meth. A320,336 (1992)

[12] J-L Biarrotte et al.., A reference accelerator scheme for ADS applications, Proc AccApp05, 656, (2006),

[13] IAEA data https://www-nds.iaea.org/sgnucdat/a 6.htm Accessed 16/3/17

[14] D Westlen and J Wallenius, Neutronic and Safety Aspects of a Gas-Cooled Subcritical Core for Minor Actinide Transmutation, Nuclear Technology 15441 (2006)

[15] J Tommasi, S Massara, S Pillon, and M Rome Minor Actinide Destruction In Dedicated Reactors, Proc. Fifth OECD/NEA Information Exchange Meeting on Actinide and Fission Product Partitioning and Transmutation, SCK-CEN, Mol, Belgium (1999) 